\title{
Fahr Disease with Seizure as Clinical Manifestation: A Case
} Series

Pauline Arantji Leyloh $^{a}$, Wardah Rahmatul Islamiyah ${ }^{\mathrm{a}, a}$

${ }^{\text {a } C o r r e s p o n d i n g ~ a u t h o r: ~ w a r d a h-r-i @ f k . u n a i r . a c . i d ~}$

${ }^{a}$ Residency Program, Department of Neurology, Faculty of Medicine, Airlangga University, Dr. Soetomo Academic General Hospital, Surabaya, Indonesia

${ }^{a}$ Staff in Department of Neurology, Faculty of Medicine, Airlangga University, Dr. Soetomo Academic General Hospital, Surabaya, Indonesia

\begin{abstract}
Brain calcification can occur physiologically and pathologically. Physiological calcification is associated with a degenerative process with globus pallidus as the most common location. Pathological calcifications are associated with several causes such as idiopathic or secondary due to disturbances in mineral metabolism in the body. Fahr disease is a syndrome characterized by calcium and phosphate deposition in several areas, namely the periventricular area, cerebellum and dentate nucleus. Fahr disease is a rare disease with an incidence of $<1: 100,000$ in the general population. In this case report, we describe 2 cases Fahr's disease both in $6^{\text {th }}$ decade of age. Both of these cases present with a clinical manifestation of seizures. The etiology behind the first case is hypoparathyroid post-thyroidectomy while the second case is idiopathic. Imaging showed bilateral calcifications of the basal ganglia and other areas such as the cerebellum and ventricles.
\end{abstract}

Keywords: Fahr disease; Intracranial Calcification; Seizures; Parathyroidism

\section{Introduction}

The process of calcification in the brain can occur physiologically and pathologically. Physiological calcification is likely due to degenerative process with globus pallidus as the most common location. Pathological calcification is associated with several causes, such as idiopathic. Other names for Fahr Disease are Idiopathic Basal Ganglia Classification or Bilateral Striopallidodentate Calcinosis is a term used to describe a neurodegenerative disease characterized by calcifications of the basal ganglia or other parts of the brain parenchyma (Batla et al., 2017) This case is rare and does not change much with a low incidence of $<1: 100,000$ population. A causal link of Fahr's disease and parathyroidism is rare. Parathyroidism is a condition that is often characterized by low serum calcium. We report 2 cases of Fahr disease in which we describe the characteristics of these cases and the treatment that should be considered when encountering such cases.

\section{Case 1}

The first patient is a 63-year-old woman with complaints of seizures for 3 times a day in September 2018. The patient had a clonic seizure as the patient jerks her extremities symmetrically and simultaneously, eyes rolling up, lasting for 1-2 minutes. During the seizure the patient was unconscious, urine leakage and 
foamy saliva. After the seizure the patient was conscious and felt weak, then the patient fell asleep after 5 hours. However, the patient experienced another seizure with the same form however the patient didn't regain consciousness followed by another seizure an hour later. The patient also complained of sudden left-sided weakness in October 2018. This complaint was accompanied by slurred speech and facial droop. Previously, the patient underwent thyroid surgery in 1985 and started to take thyroxin regularly. In 1991, the patient had her first seizure with the same form but was never treated. In 2000, the patient was declared to have recurrent hypocalcemia, hence she took 1 tablet of olcatriol regularly every day and 200mg of Carbamazepine daily. The patient said she often had seizures once every 3-4 months. The patient finally had no more seizures from 2015 - September 2018 when she experienced recurrent seizure.

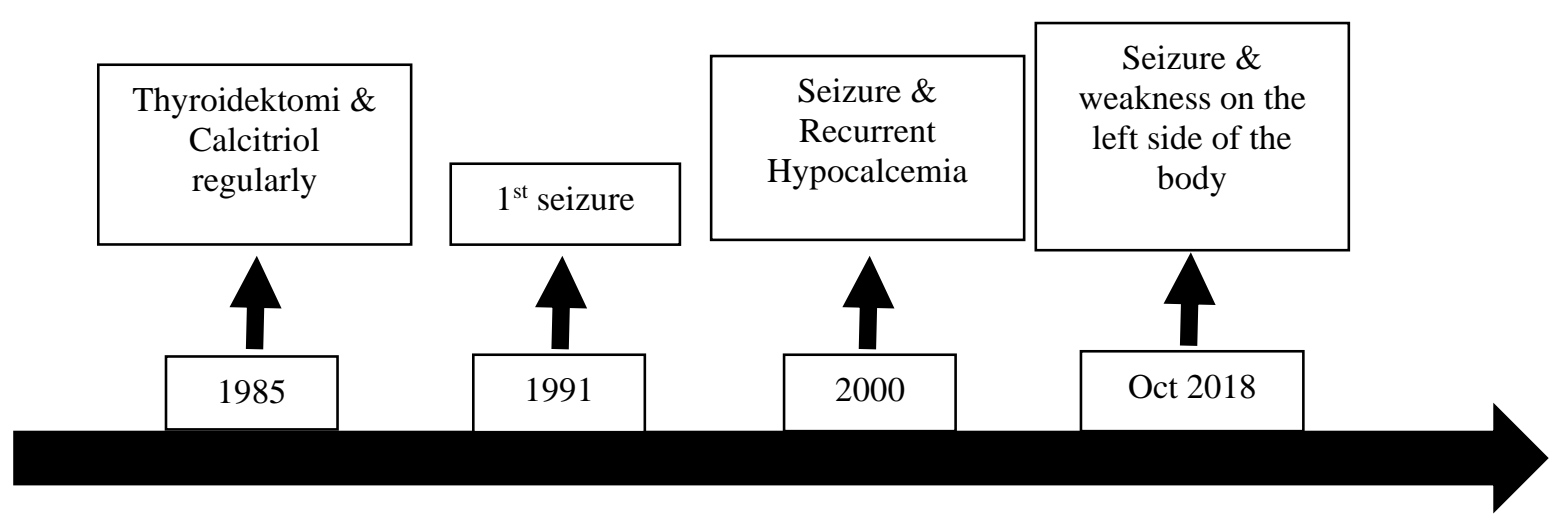

Figure 1. Time line for first case.

Head CT scan without contrast showed massive calcifications in the centrum semiovale, corona radiata, basal ganglia and right and left cerebellum. The patient did not complain of any behavioral or balance disorders. The laboratory results showed hypocalcemia of $4.8 \mathrm{mg} / \mathrm{dL}$ (normal levels $8.5-10.1 \mathrm{mg} / \mathrm{dL}$ ). Therefore, this patient underwent calcium correction through the administration of calcium gluconate. The patient also underwent two EEG examinations, the first recording showed an abnormal result, showing a subclinical serial electrographic seizure general, indicating mild diffuse encephalopathy. The carbamazepine was replaced with valproic acid tablets 500mg twice daily. After administration of valproic acid for 1 week, another EEG recording was done and showed normal result and no epileptiform activity or abnormal slowing were found. 

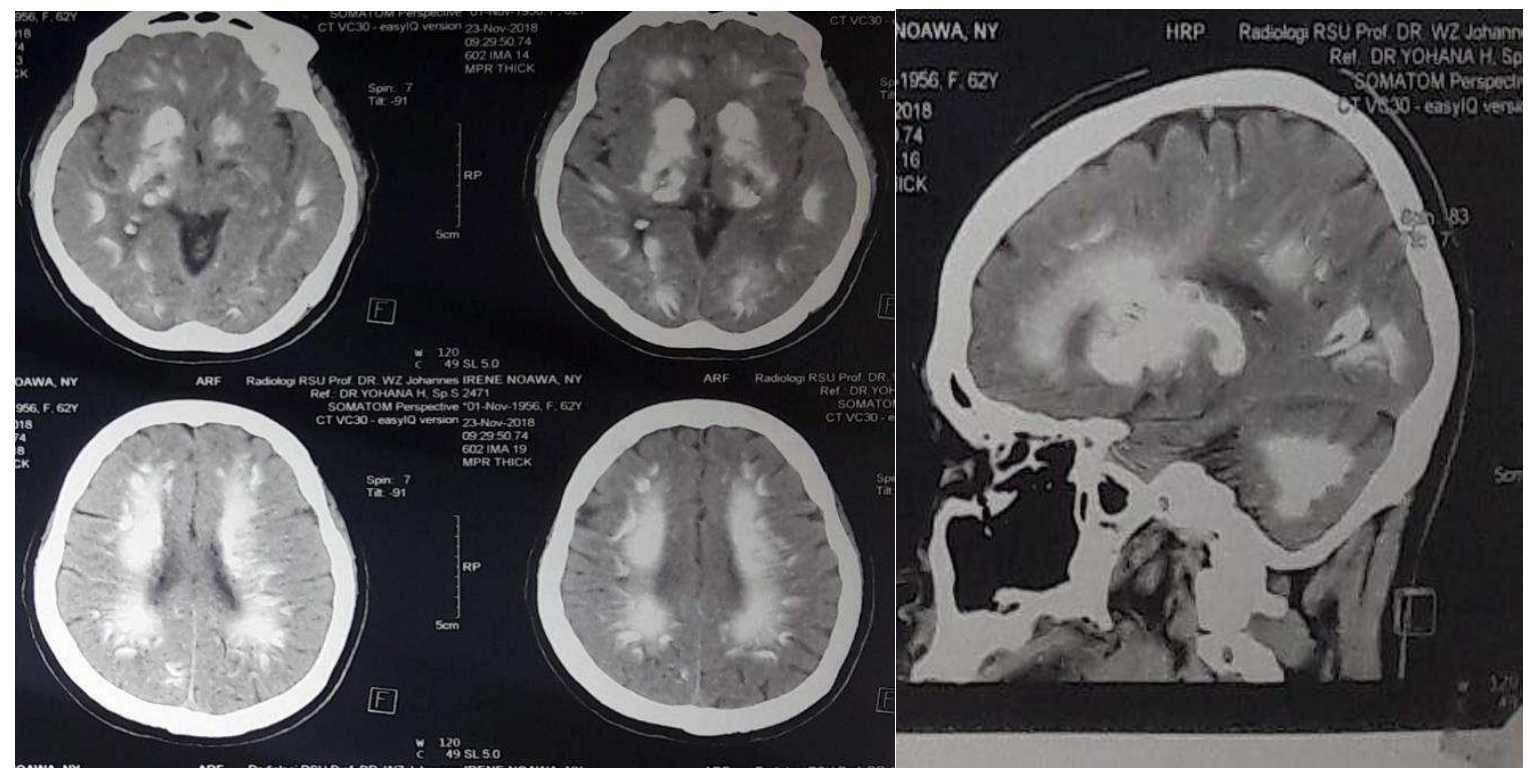

Figure 2. Sagittal and axial head CT scan without contrast in the first patient showed massive calcifications in several locations.

\section{Case 2}

The second patient is a 65-year-old woman with complaints of tremors in her left hand since October 2018, the patient also complained of clonic seizures symmetrically and simultaneously, eyes rolled up, and urine leakage the following month. Seizures last $<1$ minute, during the seizure the patient was unconscious, fortunately after the seizure the patient is conscious. There was no previous history of seizures and no other complaints, such as other movement disorders.

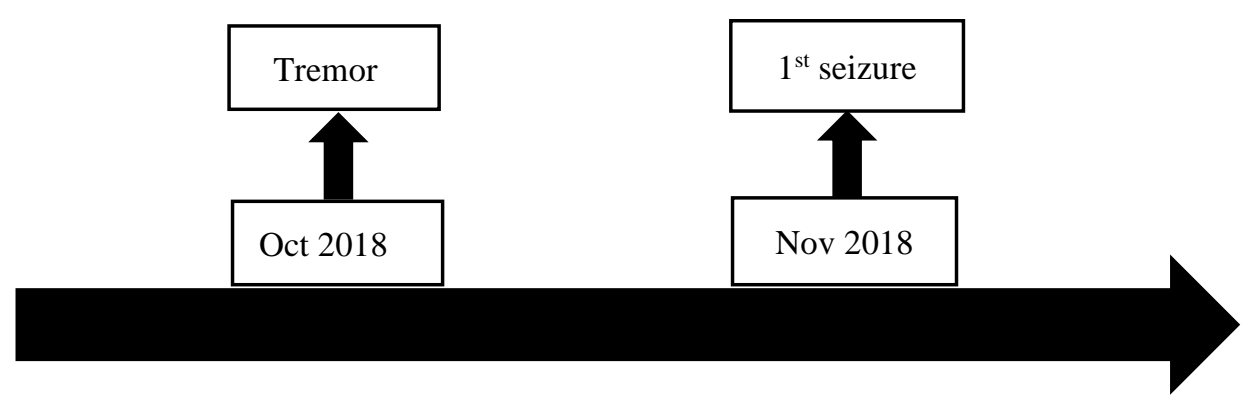

Figure 3. Time line for second case.

Head CT scan without contrast showed bilateral calcifications in the basal ganglia and cerebellum. Laboratory examination showed the patient had hypocalcemia with calcium level of $5.0 \mathrm{mg} / \mathrm{dL}$, while EEG examination showed moderate degree of generalized epileptic encephalopathy. In this patient, the patient was given with 
100mg phenytoin three times daily and hypocalcemia was treated with calcium gluconate.

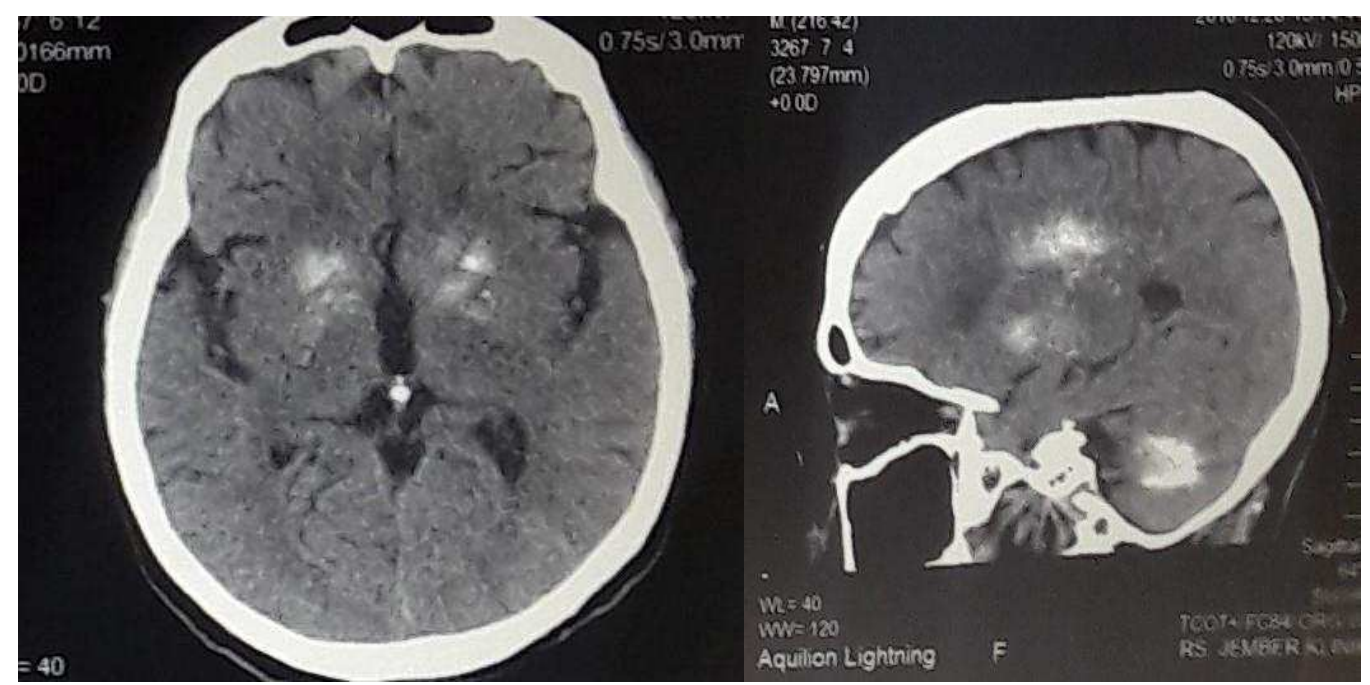

Figure 4. Calcifications in the basal ganglia and cerebellum were seen bilaterally in the second case

\section{Discussion}

The cases in this series have similar demographic characteristics as both patients are female with the onset of complaints in the $3^{\text {rd }}$ decade for the first case and $6^{\text {th }}$ decade for the second case. Current literature concludes that the incidence of Fahr disease did not different between sex and this disease with the onset of symptoms between $2^{\text {nd }}$ to $5^{\text {th }}$ decade (Batla et al., 2017).

The etiology behind the process of calcification process in the basal ganglia and bilateral striatopallidodentatum pathways are genetic factors, namely mutations on chromosome 8 , chromosome 2, chromosome 14q and SLC20A2 (Batla et al., 2017; Mufaddel \& Al-Hassani, 2014; Saleem et al., 2013) and secondary causes such as endocrine disorders (hypoparathyroidism, pseudohypoparathyroid hyperparathyroidism), infectious diseases (intrauterine and perinatal infections), and degenerative factors (Saleem et al., 2013). In our case series, no genetic examination was carried therefore we cannot conclude the relationship between Fahr's disease and genetic mutations as the primary etiology, however we tried to dig up information regarding the possibility of genetic mutations through family history in both of these cases. However, there were no family members who had the same complaint or disease. Furthermore, it was clear that in both cases, hypocalcemia was suspected due to hypoparathyroid. In the first case, the etiology of Fahr disease was hypoparathyroidism post-thyroidectomy. Thyroidectomy led to patients to hypoparathyroidism with the clinical manifestation of seizures 7 years post-thyroidectomy in the first case and hemiparesis 30 years postsurgery. However, the etiology of the second patient is still unknown. Parathyroid hormone (PTH) regulates calcium levels in the blood. If patient experienced hypocalcemia and hyperphosphatemia, it can be concluded that PTH failed to function properly. Failure of the PTH regulation can be due to congenital or acquired disease and it is an irreversible condition (Schafer \& Shoback, 2013; Sinnott, 2018).

It takes about 8-19 years to cause calcification in the basal ganglia area from chronic hypoparathyroidism alone (Brenner, 2011; Cating-Cabral \& Clarke, 2015). Several literatures suggested that 
phosphate levels $>70 \mathrm{mg} / \mathrm{dl}$ and hypocalcemia will trigger calcification in several tissues such as the skin, joints, cornea, blood vessels, and brain with the periventricular area as the site-specific predilection (Betsholtz \& Keller, 2014). There are PTH2 receptors and PTHrp receptors in the basal ganglia and cerebellum areas that mediates the calcium influx and efflux in striatum cells (Goswami et al., 2012). In addition, there is an enzyme PTH-responsive carbonic anhydrase-II (CA-II) in the basal ganglia that plays a role in the demineralization process. In addition, PTH-responsive carbonic anhydrase-II (CA-II) enzyme in the basal ganglia also plays a role in the demineralization process. Decreased CA-II enzyme will reduce $\mathrm{H}^{+}$-mediated demineralization which has a high prevalence (77\%) in Fahr disease (Goswami et al., 2012, 2014).

In this case series, both patients experienced seizure and one patient experienced movement disorder. The common clinical manifestations of Fahr disease are hyperkinetic movement disorders (chorea, tremor, dystonia, athetosis, orofacial dyskinesia), other neurological disorders (seizures, dementia, decreased consciousness) and neuropsychiatric disorders (psychosis, depression) (Abubakar \& Saidu, 2012; Batla et al., 2017; Saleem et al., 2013).

In both cases, head CT scan without contrast were used to confirm the diagnosis as previous literature used the same imaging modality. CT scan is more superior to MRI in detecting calcium deposition to depict the process of calcification (Batla et al., 2017) (Manyam et al, 1992; Batla et al., 2017). If head MRI is used as the imaging modality, the preferred sequence is susceptibility-weighted MRI as it is able to provide a low-intensity image on the T2 sequence and low or high intensity images on the T1 sequence (Batla et al., 2017; Sahin et al., 2015). The results of head CT scan in both cases showed calcification in the basal ganglia, centrum semiovale and cerebellum which were also seen in previous studies as the internal globus pallidus, cerebellum, centrum semiovale, brain stem, and subcortical white matter were the most common areas of calcification (Saleem et al., 2013; Tai, 2015). Other supporting examinations recommended to be done are serum calcium, serum phosphorus, serum magnesium, and alkaline phosphatase.

Therapeutic management of Fahr disease mainly focuses on overcoming the clinical symptoms that arise. In this case series, the first patient complained of seizures and hypocalcemia. As for the seizure, the choice of seizure medication is based on the previously performed EEG. The first patient had an abnormal EEG recording, diagnostic of a subclinical serial electrographic generalized seizure, indicating mild-grade diffuse encephalopathy. Therefore, she received $500 \mathrm{mg}$ valproic acid twice daily. As for the second patient, she had abnormal EEG recording, diagnostic of a moderate-grade diffuse generalized encephalopathy epileptic seizure. Therefore, she was given $100 \mathrm{mg}$ phenytoin three times daily. Both of these patients received calcium gluconate to correct the hypocalcemia rapidly. The first patient had a fairly long course of illness with three years of seizure-free phase, from 2015 to 2018. This can be explained by the patient's EEG recording which showed subclinical serial electrographic generalized seizure, i.e. the patient was having seizures continuously without any clinical signs or symptoms.

Seedat et al. stated that seizure is a significant predictor of calcification process and its progression in Fahr Disease.(Seedat et al., 2018) Seizure is a rare clinical manifestation of Fahr disease, most likely due to the calcification process affecting the basal ganglia and rarely the cortex. In several studies it has been reported that changes in the EEG may occur due to idiopathic or secondary hypoparathyroid conditions. Findings of sharp waves and slow waves are referred as parathyroid epilepsy. These EEG changes can occur even in the absence of clinical symptoms such as motor seizures. Other changes in EEG include abnormal background activity and associated reactions. This is associated with low levels of calcium. Correction of serum calcium level will lead to clinical improvement however results of EEG recordings will persist for several weeks or even months and may even develop into epilepsy. The cause of epilepsy in Fahr disease is related to the site of calcification but also a chronic hypokalemia resulting in increased excitability of neurons (Han et al., 2015; Modi et al., 2014; 
Nardone et al., 2016).

\section{Conclusion}

Fahr disease is a rare disease with congenital or acquired etiology that is associated with other conditions triggering the process of calcification in various areas of the brain. This case series emphasize the importance of brain imaging in seizure that first appeared during adulthood. Imaging modalities that can be used are head CT scan or head MRI with susceptibility weighted imaging sequences. If brain imaging showed bilateral calcifications, additional laboratory tests such as serum calcium levels, parathyroid hormone, thyroid hormone, serum phosphate levels, serum magnesium levels, and alkaline phosphate level should be done to rule in or out the suspicion of Fahr disease. Therefore, patients are able to receive appropriate therapeutic management promptly to prevent other clinical manifestations and disease progression.

\section{References}

Abubakar, S. A., \& Saidu, S. (2012). Idiopathic bilateral strio-pallido-dentate calcinosis (Fahr's disease): A case report and review of the literature. Annals of African Medicine, 11(4), 234-237. https://doi.org/10.4103/1596-3519.102855

Batla, A., Tai, X. Y., Schottlaender, L., Erro, R., Balint, B., \& Bhatia, K. P. (2017). Deconstructing Fahr's disease/syndrome of brain calcification in the era of new genes. Parkinsonism and Related Disorders, 37, 1-10. https://doi.org/10.1016/j.parkreldis.2016.12.024

Betsholtz, C., \& Keller, A. (2014). PDGF, pericytes and the pathogenesis of idiopathic basal ganglia calcification (IBGC). Brain Pathology, 24(4), 387-395. https://doi.org/10.1111/bpa.12158

Brenner. (2011). Disorders of Calcium, Magnesium, and Phosphate Balance. Pocket Companion to Brenner and Rector's The Kidney, 166-185. https://doi.org/10.1016/b978-1-4160-6640-8.00008-7

Cating-Cabral, M. T. B., \& Clarke, B. L. (2015). Epidemiology of Hypoparathyroidism. In The Parathyroids: Basic and Clinical Concepts: Third Edition (Third Edit). Elsevier Inc. https://doi.org/10.1016/B978-0-12-397166-1.00049-7

Goswami, R., Millo, T., Mishra, S., Das, M., Kapoor, M., Tomar, N., Saha, S., Roy, T. S., \& Sreenivas, V. (2014). Expression of osteogenic molecules in the caudate nucleus and gray matter and their potential relevance for basal ganglia calcification in hypoparathyroidism. Journal of Clinical Endocrinology and Metabolism, 99(5), 1741-1748. https://doi.org/10.1210/jc.2013-3863

Goswami, R., Sharma, R., Sreenivas, V., Gupta, N., Ganapathy, A., \& Das, S. (2012). Prevalence and progression of basal ganglia calcification and its pathogenic mechanism in patients with idiopathic hypoparathyroidism. Clinical Endocrinology, 77(2), 200-206. https://doi.org/10.1111/j.1365-2265.2012.04353.x

Han, P., Trinidad, B. J., \& Shi, J. (2015). Hypocalcemia-induced seizure: Demystifying the calcium paradox. ASN Neuro, 7(2), 1-9. https://doi.org/10.1177/1759091415578050

Modi, S., Tripathi, M., Saha, S., \& Goswami, R. (2014). Seizures in patients with idiopathic hypoparathyroidism: Effect of antiepileptic drug withdrawal on recurrence of seizures and serum calcium control. European Journal of Endocrinology, 170(5), 777-783. https://doi.org/10.1530/EJE-14-0075

Mufaddel, A. A., \& Al-Hassani, G. A. (2014). Familial idiopathic basal ganglia calcification (Fahr's disease). Neurosciences, 19(3), 171-177.

Nardone, R., Brigo, F., \& Trinka, E. (2016). Acute symptomatic seizures caused by electrolyte disturbances. Journal of Clinical Neurology (Korea), 12(1), 21-33. https://doi.org/10.3988/jcn.2016.12.1.21

Sahin, N., Solak, A., Genc, B., \& Kulu, U. (2015). Fahr disease: use of susceptibility-weighted imaging for diagnostic dilemma with magnetic resonance imaging. Quantitative Imaging in Medicine and Surgery, 5(4), 628-632. https://doi.org/10.3978/j.issn.2223-4292.2015.04.01

Saleem, S., Aslam, H. M., Anwar, M., Anwar, S., Saleem, M., Saleem, A., \& Rehmani, M. A. K. (2013). Fahr's syndrome: Literature review of current evidence. Orphanet Journal of Rare Diseases, 8(1), 1. https://doi.org/10.1186/1750$1172-8-156$

Schafer, A. L., \& Shoback, D. (2013). Hypocalcemia : Defi nition, Etiology, Pathogenesis, Diagnosis , and Management. 572-578.

Seedat, F., Daya, R., \& Bhana, S. A. (2018). Hypoparathyroidism Causing Seizures: When Epilepsy Does Not Fit. Case 
Reports in Medicine, 2018(Figure 3). https://doi.org/10.1155/2018/5948254

Sinnott, B. P. (2018). Hypoparathyroidism - Review of the Literature 2018. Journal of Rare Disorders: Diagnosis \& Therapy, 04(03), 1-7. https://doi.org/10.21767/2380-7245.100180

Tai, X. Y. (2015). Fahr' 's disease : current perspectives. 43-49. 